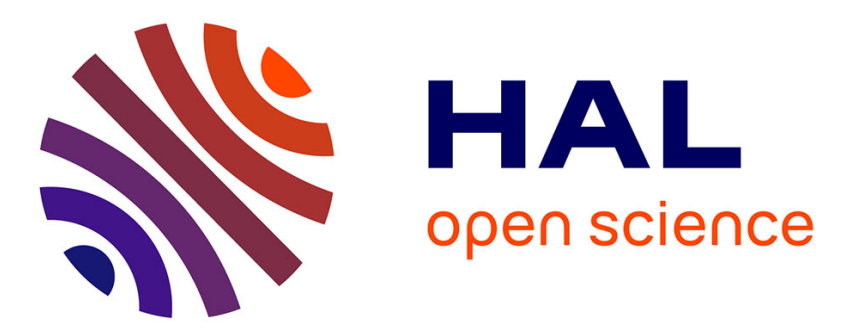

\title{
Dynamic Interfacial Tension Effects in the Rupture of Liquid Necks
}

Matthieu Robert de Saint Vincent, Julien Petit, Mounir Aytouna, Jean-Pierre Delville, Daniel Bonn, Hamid Kellay

\section{- To cite this version:}

Matthieu Robert de Saint Vincent, Julien Petit, Mounir Aytouna, Jean-Pierre Delville, Daniel Bonn, et al.. Dynamic Interfacial Tension Effects in the Rupture of Liquid Necks. Journal of Fluid Mechanics, 2012, 692, pp.499-510. 10.1017/jfm.2011.550 . hal-00677245

\section{HAL Id: hal-00677245 \\ https://hal.science/hal-00677245}

Submitted on 15 Dec 2017

HAL is a multi-disciplinary open access archive for the deposit and dissemination of scientific research documents, whether they are published or not. The documents may come from teaching and research institutions in France or abroad, or from public or private research centers.
L'archive ouverte pluridisciplinaire HAL, est destinée au dépôt et à la diffusion de documents scientifiques de niveau recherche, publiés ou non, émanant des établissements d'enseignement et de recherche français ou étrangers, des laboratoires publics ou privés.

\section{다(1)(2)}

Distributed under a Creative Commons Attribution - ShareAlikel 4.0 International 


\title{
Dynamic interfacial tension effects in the rupture of liquid necks
}

\author{
M. Robert de Saint Vincent ${ }^{1}$, J. Petit ${ }^{1}$, M. Aytouna ${ }^{2}$, J. P. Delville ${ }^{1}$, \\ D. Bonn ${ }^{2,3}$ and H. Kellay ${ }^{1} \dagger$ \\ ${ }^{1}$ Laboratoire Ondes et Matière d'Aquitaine (UMR 5798 CNRS), U. Bordeaux 1, 351 cours de la \\ Libération, 33405 Talence, France \\ 2 Van Der Waals-Zeeman Institute, University of Amsterdam, Valckenierstraat 65, \\ 1018XE Amsterdam, The Netherlands \\ ${ }^{3}$ Laboratoire de Physique Statistique de l'ENS, 24 rue Lhomond, 75005 Paris, France
}

\begin{abstract}
By examining the rupture of fluid necks during droplet formation of surfactant-laden liquids, we observe deviations from expected behaviour for the pinch-off of such necks. We suggest that these deviations are due to the presence of a dynamic (timevarying) interfacial tension at the minimum neck location and extract this quantity from our measurements on a variety of systems. The presence of such dynamic interfacial tension effects should change the rupture process drastically. However, our measurements show that a simple ansatz, which incorporates the temporal change of the interfacial tension, allows us to understand the dynamics of thinning. This shows that this dynamics is largely independent of the exact details of what happens far from the breakup location, pointing to the local nature of the thinning dynamics.
\end{abstract}

Keywords: breakup/coalescence, liquid bridges

\section{Introduction}

Droplet formation is needed for a variety of processes such as the formation of emulsions, inkjet printing, as well as microfluidic applications. In many of these instances, surface-active material is present. When drop formation from such surfactant-laden solutions is considered, the detailed dynamics of the rupture process leading to drop formation and eventually to satellite drops will be affected by the properties of the surface (Eggers 1997; Eggers \& Villermaux 2008). Several studies have focused on drop formation in the presence of insoluble surfactants (Ambravaneswaran \& Basaran 1999; Craster, Matar \& Papageorgiou 2002; Timmermans \& Lister 2002; Liao, Franses \& Basaran 2006; McGough \& Basaran 2006; Xu, Liao \& Basaran 2007). Fewer studies have been devoted to soluble surfactants (Jin, Gupta \& Stebe 2006; Craster, Matar \& Papageorgiou 2009). In both cases, the surfactant surface concentration in the neck region may change significantly during the rupture process, engendering Marangoni stresses that affect the size of the satellite drops (Craster et al. 2009) and the shape of the neck connecting the drop

$†$ Email address for correspondence: hamid.kellay@u-bordeaux1.fr 
to the fluid reservoir (Liao et al. 2004; Jin et al. 2006). Theoretical work on drop formation with insoluble surfactants suggests that the ultimate stages of the thinning of the fluid neck just before breakup may be accompanied by a total depletion of the surfactants from the rupture zone leaving the surface bare, i.e. without surfactant (Timmermans \& Lister 2002). That the surfactant is depleted significantly from the rupture zone, engendering large gradients of the surface concentration of the surfactant has been confirmed by numerical work for both soluble (Craster et al. 2009) and insoluble surfactants (Craster et al. 2002; Xu et al. 2007). The thinning of the neck engenders large stretching rates of the surface area so that surfactants have no time to either diffuse over the interface or to arrive from the bulk to saturate the interface in the thinning region. It is in this respect that soluble and insoluble surfactants differ: in the former both surface diffusion and replenishment of the surface by surfactants from the bulk are allowed, with a non-trivial relation between bulk surfactant concentration and surface tension; while in the latter surfactant redistribution occurs only through diffusion along the surface. In practice, experiments using insoluble surfactants are difficult to carry out but recent experimental work using soluble surfactants showed that the thinning dynamics is indeed affected by their presence and that the effective interfacial tension at the neck location may increase in time as the liquid thread tends to break up (Roché et al. 2009). This increase is directly related to the change of surface coverage by the surfactant, in agreement with previous numerical work for soluble surfactants (Craster et al. 2009).

Here we examine several cases of the dynamics of liquid bridge rupture going from the fast inertial thinning regime all the way to the viscous-dominated regime to explore the rupture process in the presence of soluble surfactants in the most complete experimental study to date. Our results highlight the role of surface-active agents in pinch-off dynamics in a variety of cases including a set of surfactants with different adsorption and desorption dynamics, the case of interfaces of microemulsions where the exchange of surfactant between the bulk fluid and the interface is different from the case of micellar solutions, and the case of surfactant mixtures. To analyse these measurements we make use of known solutions for the thinning of the neck of bare interfaces (i.e. in the absence of surfactants). We then introduce a time-dependent surface tension to account for departures from such behaviour. The rationale behind this time-varying surface tension is the possible depletion of surfactant from the pinch-off region as found in numerics (Craster et al. 2009). This procedure is similar to that used for the pinch-off dynamics of a class of complex fluids for which the viscosity depends on the deformation rate and therefore on time (Doshi et al. 2003; Renardy \& Renardy 2004; Savage et al. 2010). The conclusion from the large set of experiments presented here is that the thinning dynamics may be understood based solely on the local interfacial tension at the neck location, pointing to the local nature of the thinning process. This procedure therefore does not shed light on other effects, which are not local, such as satellite formation (Craster et al. 2009).

\section{Brief overview of pinch-off dynamics in the inertial and viscous regimes}

The thinning dynamics of a rupturing fluid neck during drop detachment is governed by different quantities such as the viscosity, the density and the interfacial tension of the interface. When viscous effects can be neglected, the dynamics of the neck thinning is said to be in the inertial range where only interfacial tension and density play a role. The radius of the neck then follows the well-known law: $R(t)=0.35(\gamma / \rho)^{1 / 3}\left(t_{c}-t\right)^{2 / 3}$ (Eggers 1997; Eggers \& Villermaux 2008). Here $\gamma$ is 
the interfacial tension, $\rho$ is the density of the liquid, $t$ is time and $t_{c}$ is the time at which the fluid neck breaks. In the opposite limit of small inertial forces, viscous effects are the dominant force resisting the motion, and the thinning dynamics obeys a linear thinning law given by $R(t)=C(\gamma / \eta)\left(t_{c}-t\right)$, where $\eta$ is the shear viscosity of the fluid undergoing pinch-off. Note that this equation is obeyed for length scales smaller than the so-called viscous length given by: $l_{v}=\left(\eta^{2} / \rho \gamma\right)$ (Eggers 1997). The constant $C$ takes a value of 0.07 (Papageorgiou 1995), for the case where the Reynolds number is negligible, a regime known as visco-capillary breakup. For later stages in the thinning process, the velocity of the fluid at the neck increases and the assumption of negligible Reynolds number is no longer valid. A new regime then sets in, known as visco-capillary-inertial breakup (Eggers 1993). In this case the thinning is still linear in time, but with $C=0.03$. The characterization as well as the transition between these two regimes has been documented in Rothert, Richter \& Rehberg (2001).

When, in addition, the breakup of the fluid occurs in an outer fluid of nonnegligible viscosity the pinch-off dynamics also changes. Consider a fluid of viscosity $\eta_{2}$ rupturing in an external fluid of viscosity $\eta_{1}$. Breakup may enter the so-called Stokes regime of two-fluid pinch-off when the scales of interest are of the order $\left(\eta_{1} / \eta_{2}\right) l_{v}$ (Lister \& Stone 1998). The thinning of the minimum neck radius in this case follows the scaling law $R(t)=V\left(t_{c}-t\right)$ where the thinning velocity is given by $V=H\left(\eta_{2} / \eta_{1}\right) \gamma / \eta_{1}$. The function $H\left(\eta_{2} / \eta_{1}\right)$ has been measured experimentally and partially understood theoretically (Lister \& Stone 1998; Cohen et al. 1999; Zhang \& Lister 1999; Cohen \& Nagel 2001).

\section{Experimental systems used}

The systems studied include: (i) water containing surfactant (we study three different types), pinching in air; (ii) water pinching in an oil phase, the latter containing a surfactant allowing ultralow interfacial tensions; and (iii) water pinching in oil, with both phases containing a different surfactant. The capillaries used for system (i) have an internal diameter of $1.95 \mathrm{~mm}$. The droplets were released from syringe-pump-fed glass capillaries of $200 \mu \mathrm{m}$ in internal diameter and $330 \mu \mathrm{m}$ external diameter for systems (ii) and (iii). System (iii) was also studied in a microfluidic device where breakup was induced in a junction producing droplets or forced through the use of a laser beam to break up a liquid jet. All surfactant solutions are sufficiently dilute that changes of their density or viscosity or the presence of visco-elastic effects that can profoundly alter the breakup (Amarouchene et al. 2001; Wagner et al. 2005; Savage et al. 2010) can be neglected.

Our measurements were based on imaging the rupture process using a high-speed camera working at rates which were adapted to the situation studied. The imaging system uses microscope objectives of different magnifications. The resulting pictures were analysed using an in-house program to extract the minimum neck radius versus time for the different systems studied. High surface tensions were measured using the drop weight technique; low interfacial tensions were measured using a spinning drop tensiometer or the surface deformation due to the radiation pressure of a laser beam focused at the surface (Mitani \& Sakai 2002).

Figure 1 shows pictures of detaching drops for our different systems. In the photographs of figure 1(a), and from top to bottom, different snapshots taken at different times from the rupture point are shown for pure water, and solutions of Heliosol, SDS, and Silwet surfactant. Note that the shape of the neck hardly changes; however, and as we will see below, the thinning dynamics is different for each case. 
(a)

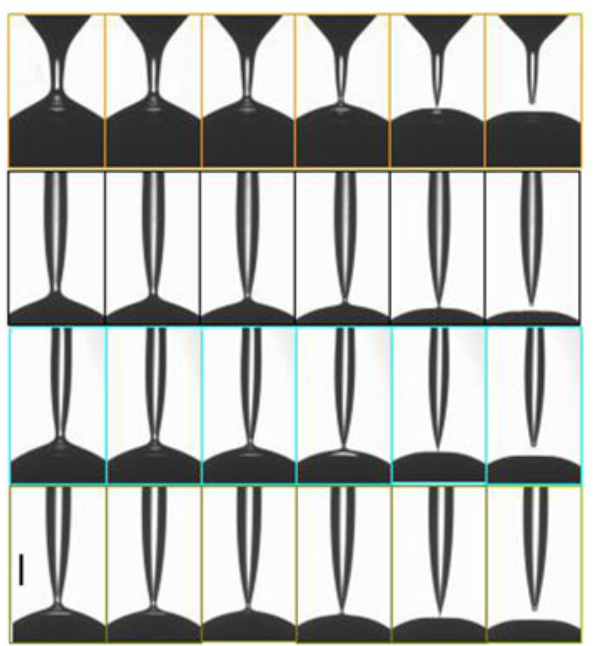

(b)

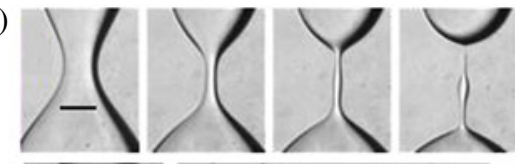

(c)

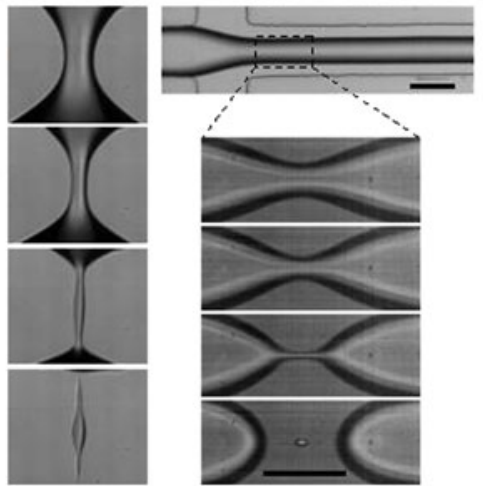

FIGURE 1. (Colour online available at journals.cambridge.org/flm) (a) Water and surfactant solutions (from top to bottom: water, Heliosol, SDS, and Silwet solutions); the images at the left are taken $0.25 \mathrm{~ms}$ before rupture and the successive images are $0.05 \mathrm{~ms}$ apart. The line is 300 microns. (b) Microemulsion system (the line is 100 microns); the images are at 7, 2.7, 1.4 and $0 \mathrm{~ms}$ before rupture. (c) Two surfactant systems and different ways to induce breakup: dripping from a capillary (left, same scale as $b$ with $26 \mathrm{~ms}$ between images) and jet breakup in a microchannel (right, the upper and lower lines are 100 and 50 microns; the images are $13 \mathrm{~ms}$ apart.

Figure 1(b) shows snapshots at different instants for the microemulsion system. Note that the final instants show the formation of a satellite drop in the neck region. The third system is shown in figure 1(c). The left panel shows a drop falling from a capillary while the right panel shows the rupture of a jet in a microchannel. Both systems show the formation of a satellite drop. Our different systems allow us to explore the rupture in the inertial and viscous regimes. In one of the systems, the inertial and viscous regimes are obtained in a single experiment. We here focus on the pinching dynamics for the three cases considered; the formation of satellite drops and their properties will not be discussed.

\section{Inertial case}

This case is represented by the rupture of a low-viscosity fluid (pure water and water plus surfactant). Three different surfactants at a concentration of $0.5 \%$ by weight were used (SDS, Silwet and Heliosol). This concentration corresponds to roughly 2 c.m.c. (critical micellar concentration) so the surface is fully covered by the surfaceactive agents while the rest of the surfactant resides in the bulk in the form of micelles and monomers. The evolution of the minimum radius of the neck is shown in figure 2(a) along with fits to the $2 / 3$ law using the equilibrium surface tension. The evolution is clearly nonlinear: it follows the $2 / 3$ law for water but shows deviations for the surfactant solutions near breakup. Variation of the breakup time $t_{c}$ can make the fit work better for the small radii in the surfactant case but at the expense of using a higher surface tension. In order to resolve this discrepancy it is useful to represent the data so that the sensitivity to the value of $t_{c}$ is removed. This is illustrated by the following analysis. Since the neck thinning dynamics should obey the inertial-range 
prediction, $R^{3 / 2}(t)$ should be a linear function of $\left(t_{c}-t\right)$ with a slope $(0.35)^{3 / 2}(\gamma / \rho)^{1 / 2}$. Note that in this representation the value of $t_{c}$ is the intercept at $R=0$. Indeed, when $R^{3 / 2}(t)$ is plotted versus $t$ (figure $2 b$ ), the pure water case shows a straight line in this representation. From the slope of the straight line in figure $2(b)$, we find $\gamma=78 \mathrm{mN} \mathrm{m}^{-1}$ which is close to the expected value of $72 \mathrm{mN} \mathrm{m}^{-1}$ for pure water. The case of surfactant-laden water is different. Here, the variation of $R^{3 / 2}(t)$ is linear at first but the slope of this linear variation increases as the rupture point is approached. If we analyse the initial dynamics (the first slope), we find interfacial tensions of 20, 34 and $35 \mathrm{mN} \mathrm{m}^{-1}$ for Silwet, SDS, and Heliosol surfactants respectively. These values are roughly consistent with the equilibrium tensions measured using the drop weight method: 25,40 and $35 \mathrm{mN} \mathrm{m}^{-1}$ for these surfactants.

The final slope of the $R^{3 / 2}(t)$ curves near the rupture point is higher than the initial slope, giving interfacial tension values of 29,49 and $56 \mathrm{mN} \mathrm{m}^{-1}$ for the same series of surfactants. Thus, near the rupture, the interfacial tension is larger than its equilibrium value (as already shown in figure $2 a$ where the fits work well far from the rupture point but not close to it). The variation of the interfacial tension versus time can be estimated from the local slope of $R^{3 / 2}(t)$ by assuming that the inertial breakup law holds locally. In accordance with the increase in slope of figure $2(b)$, the interfacial tension, inset of figure $2(b)$, starts near its measured equilibrium value before increasing by roughly a factor of two as $t_{c}$ is approached. This holds for the three surfactants used. The fact that the initial surface tension is close to its equilibrium value initially and that it increases as rupture is approached is consistent with surfactant depletion at the pinch-off location as found in numerics.

The relative increase in tension is roughly similar for the three different surfactants despite the fact that the dynamics of these surfactants is supposed to be different from case to case. A priori, the Silwet surfactant is supposed to have slower adsorption dynamics than say SDS, while the Heliosol surfactant is supposed to have the fastest dynamics (Aytouna et al. 2010). We believe that such differences may not be relevant to the present case since in the rupture case, the surface starts covered, or at least partially covered, then through the stretching, the surface area increases leading to a decrease of the coverage by the surfactants. In classical dynamic interfacial tension measurements (Christov et al. 2006) from which surfactant adsorption dynamics can be obtained, the contrary occurs, i.e. the surface starts without surfactant and in time, the coverage increases.

It is worthwhile noting that, in disagreement with recent theoretical predictions (Timmermans \& Lister 2002), albeit for insoluble surfactants, the final interfacial tension is not that of pure water. In principle, it remains possible that there is an ultimate state of breakup in which the surface is completely devoid of surfactants. Our results show that if this happens it would occur when the radius is below our spatial resolution, which is one micrometre. We believe that such a situation may occur for low surfactant concentrations below the c.m.c. To test this possibility, additional experiments at a concentration of $0.05 \%$ were carried out. The result, displayed in figure 2(c), shows that the interfacial tensions extracted from a similar analysis as in figure $2(b)$, are roughly constant over most of the time span of the rupture. In addition, the interfacial tension, near pinch-off (see inset of figure $2 c$ ), is roughly that of water as indicated by the black line. The results for pure water are also included in this figure. Within experimental error, the slopes near the rupture point indicate that the interface is practically devoid of surfactant. The low concentrations therefore show an important depletion of surfactant at the rupture point and that for the three surfactants used. 

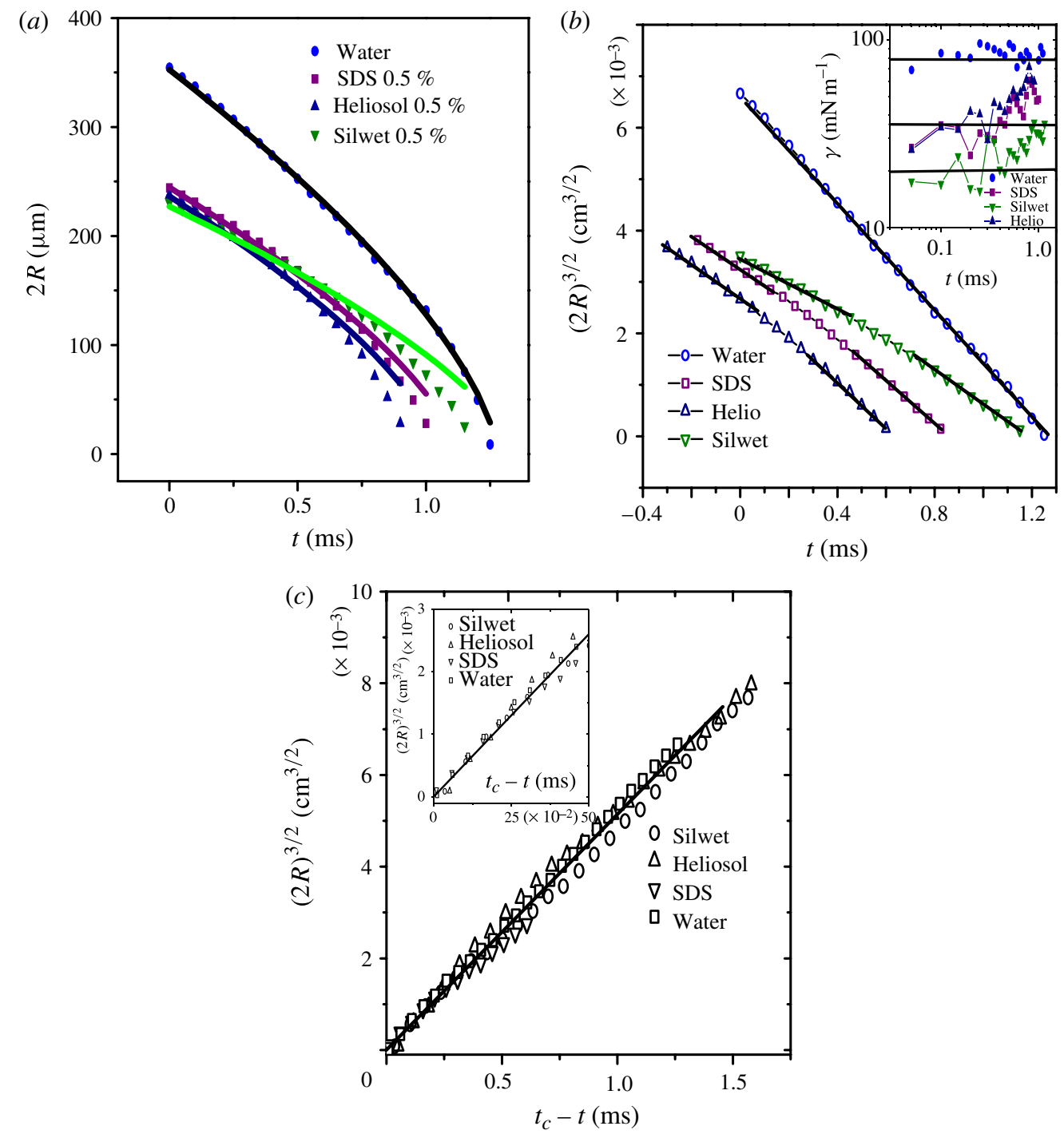

FIgURE 2. (Colour online) (a) Thinning dynamics for water and the different surfactant solutions. The solid lines are fits using the $2 / 3$ power law and known interfacial tension at equilibrium. $(b)$ Expected linear dependence of $R^{3 / 2}(t)$. Note that in the presence of surfactants, the linear behaviour is valid at the begining and near the end with a crossover at intermediate times. The SDS and Heliosol case were shifted by -0.175 and $-0.3 \mathrm{~ms}$ for visibility. Inset: extracted interfacial tension versus time. The horizontal lines represent the measured value at equilibrium. (c) Thinning dynamics for the case of a low surfactant concentration. The final instants before rupture (see inset) show a behaviour similar to that of the bare interface, i.e. water. The solid lines indicate the expected thinning dynamics for water.

\section{Two-fluid pinch-off and viscous pinch-off}

System (ii) is a microemulsion obtained from a water-heptane-AOT mixture at $40 \mathrm{mM}$ AOT (bis-ethyl-hexyl-sulphosuccinate surfactant) and $0.055 \mathrm{M}$ salt in water (Binks et al. 2000). The interface between the oil phase (which is rich in AOT) and 


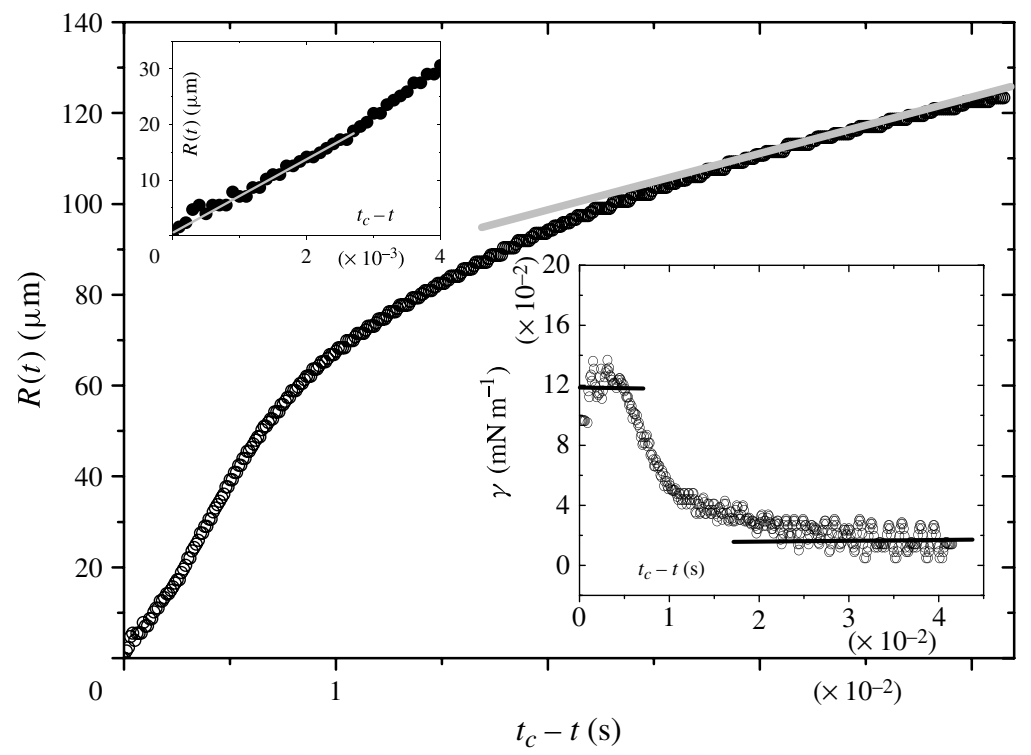

FIGURE 3. Thinning dynamics in the microemulsion system where water drops are formed in oil. The thinning dynamics is expected to be linear and in the two-fluid Stokes regime below $30 \mu \mathrm{m}$. Above this scale the dynamics should be in the viscous regime. The grey line in the main figure is the expected variation using the equilibrium surface tension. The upper inset shows the final instants of the thinning where a linear regime is observed. Lower inset: surface tension versus time. The horizontal lines indicate the initial and the final surface tension.

the water has an ultralow tension of the order of $10 \mu \mathrm{N} \mathrm{m}^{-1}$. Our own measurements of the surface deformation by a laser beam (Mitani \& Sakai 2002) and a spinning drop technique give values of 13 and $15 \mu \mathrm{N} \mathrm{m}^{-1}$, respectively, which is consistent with previous measurements (Binks et al. 2000). This system has a viscous length scale of the order of $70 \pm 5 \mu \mathrm{m}$. Because of the presence of the external fluid of non-negligible viscosity, the thinning dynamics should be in the two-fluid Stokes regime. This regime occurs for length scales below $\eta_{1} / \eta_{2} l_{v}\left(\eta_{1}=0.37 c P\right.$ for the outer fluid and $\eta_{2}=1 \mathrm{cP}$ for the inner fluid) which is near $26 \pm 2 \mu \mathrm{m}$. The thinning of the minimum neck radius should therefore be linear in time with $H=0.02$ for this combination of fluids. The thinning curve for this case is shown in figure 3, with a close-up view of the last instants before neck breakup in the upper inset.

Two different regimes are present: a slow thinning regime at early times and an accelerated thinning regime near pinch off. The expected thinning dynamics (using the equilibrium surface tension and the visco-capillary scaling) seems to be valid at early times as indicated by the grey line in figure 3. As rupture is approached, the thinning dynamics deviates from the expected result and shows an important acceleration. If we now focus on the final instances before pinch-off, the dynamics should be approximated correctly using the linear thinning law for two-fluid pinch-off (see upper inset of figure 3). The value of the surface tension obtained from this analysis is near $120 \mu \mathrm{N} \mathrm{m}$. This surface tension is much higher than its equilibrium value. Because of the high value of the tension, there may be an ambiguity as to whether this regime is described by the two-fluid Stokes law or by the visco-capillary law; however, the use of the visco-capillary scaling with $C=0.07$ also yields a high 
value, close to the previous one, of about $100 \mu \mathrm{N} \mathrm{m}$. This indicates that in the last instants before breakup, and even though the linear asymptotic solution seems to hold, the surface is not at equilibrium suggesting again that surfactant is depleted from the pinch-off location. If we consider that the slope of the variation of $R$ versus time is given by a local interfacial tension, this quantity can be estimated and plotted versus time as in the previous example. This tension, see the lower inset of figure 3, starts out close to its equilibrium value (indicated by the lower horizontal line) at early times before pinch-off and increases as the pinch-off time is approached towards a value close to that determined using the final linear regime (upper horizontal line). That the early time dynamics gives a surface tension close to the equilibrium value justifies $a$ posteriori our use of the visco-capillary regime dynamics even though the scales are near or slightly higher than the expected viscous length scale. This system therefore shows a complex behaviour but one which is similar to other surfactant systems: an increase of the surface tension as pinch-off is approached. This is so despite the fact that the mixture is a microemulsion and therefore the rules of surfactant exchange between the bulk of the fluid and the interface are different from usual micellar solutions.

\section{Two-fluid pinch-off and inertial pinch-off}

The third case under study uses water and hexadecane and two surfactants: SDS is added at $2.5 \%$ in weight (10 c.m.c.) in water and Span 80 at $0.3 \%$ in weight ( $\simeq 5$ c.m.c.) in oil. First we measured the thinning rate of the neck of a detaching water drop, falling from a capillary under the action of gravity, in oil. The equilibrium surface tension for this system being near $0.1 \mathrm{mN} \mathrm{m}^{-1}$, the viscous length scale is near $10 \mu \mathrm{m}$ and the scale for which the two-fluid Stokes regime should be observed is near $30 \mu \mathrm{m}$. The thinning dynamics is shown in figure $4(a)$. We note that the final instants before rupture at neck radii below $\sim 20 \mu \mathrm{m}$ can be described quite well by a linear law. This is shown for two different runs in the inset of figure 4(a). By using the linear law quoted above for two-fluid pinch-off, and the appropriate $H$ value of 0.06 (Cohen \& Nagel 2001), we deduce an interfacial tension for the final instants of 1.1 and $1.7 \mathrm{mN} \mathrm{m}^{-1}$ for the two runs presented. The slight difference between these two values is probably due to the initial conditions and further interfacial rearrangements when two surfactants - one ionic, SDS, and one non-ionic, Span 80 - are suddenly put in contact at the tip of the capillary. Again and while the asymptotic solution seems to hold, the surface appears to be far from equilibrium since the estimated surface tension is much higher than its equilibrium value. Since the viscous length scale for this system is quite low, the dynamics for larger radii should be in the inertial regime as neither the visco-capillary nor the inertial visco-capillary regimes are possible.

By analysing the data for early times in a similar way as above in the inertial regime we can estimate the variation of the interfacial tension versus time using the local slope of $R^{3 / 2}(t)$, which is shown in the inset of figure $4(b)$. The black line in the inset at early times shows the expected dynamics using a surface tension of $0.1 \mathrm{mN} \mathrm{m}^{-1}$. The variation of the interfacial tension versus time is shown in figure $4(b)$. This tension starts near $0.1 \mathrm{mN} \mathrm{m}^{-1}$ which is close to the equilibrium value with the two runs giving roughly similar initial tensions. Both runs also show an increase of the tension versus time which goes through a mild maximum before reaching a value close to that deduced from the linear regime near pinch-off. Here again and despite the presence of the inertial regime followed by a two-fluid Stokes 

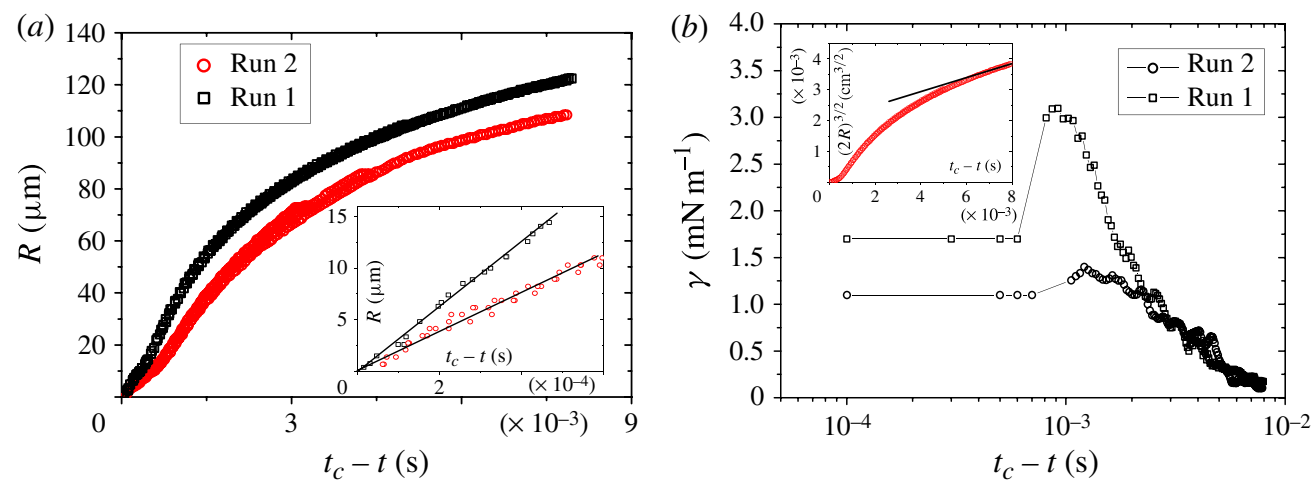

Figure 4. (Colour online) (a) Thinning dynamics of water in oil in the presence of two surfactants. The thinning shows two regimes with a linear regime near pinch-off (inset). $(b)$ The inset shows $R^{3 / 2}(t)$ versus time for the early time regime. The variation while close to linear initially (indicated by the black line), is not linear throughout as expected, signalling possible changes in interfacial tension. The main figure shows the interfacial tension extracted from the slope of the $R^{3 / 2}(t)$ curve. Two different runs are shown. The interfacial tension shows an increase as the rupture is approached.

regime, the time variation of the interfacial tension may be sufficient to explain the complex thinning dynamics.

This same system was also used in a microfluidic channel to study the breakup of drops of water in oil (Delville et al. 2009). Here the breakup is driven by a co-flow in a cross-shaped junction as shown in figure 1(c). This channel is made of PDMS, cast on a glass slide, and has a rectangular $50 \mu \mathrm{m} \times 100 \mu \mathrm{m}$ cross-section. Water and oil are injected at constant flow rates using syringe pumps. The two liquids are injected without prior equilibration. The cross-shaped junction allows for the formation of small water drops. The dynamics of neck thinning during drop formation is shown in the inset of figure 5. The neck thins slowly at first with a dynamics that is well approximated by a linear law before accelerating at the final instants before rupture. By analysing the data using the two-fluid pinch-off solution for neck radii smaller than $30 \mu \mathrm{m}$, we deduce an interfacial tension which starts low (near $0.1 \mathrm{mN} \mathrm{m}^{-1}$ as obtained from the initial variation of the radius versus time approximated as linear (see inset)) and increases up to $2 \mathrm{mN} \mathrm{m}^{-1}$ as shown in figure 5, a value in agreement with the ones deduced at the end of the breakup of water drops falling in oil (figure $4 b$ ). Here also, the early dynamics is given by the equilibrium surface tension while the later stages show a significantly higher tension.

In a different experiment, we produced a jet of water in such a co-flow cell by using a higher water injection rate. This jet is destabilized by focusing, for a brief moment, a laser beam near the jet. A dye (commercial E122 food dye) was added to the water to favour light absorption and locally heat up the water jet. This produces thermocapillary stresses and forces pinching when the beam power exceeds a threshold (Delville et al. 2009). By studying the rupture of the jet, after the laser has been turned off, we obtain its thinning dynamics under such conditions. Here again, an analysis of the variation of $R$ versus time (see inset of figure 5) using the two-fluid pinch-off dynamics allows us to deduce an interfacial tension during pinch-off. The temporal variation of the effective tension from the two experiments (i.e. with and without the use of a laser beam) is compared in figure 5. Note that this tension increases by over an order of 


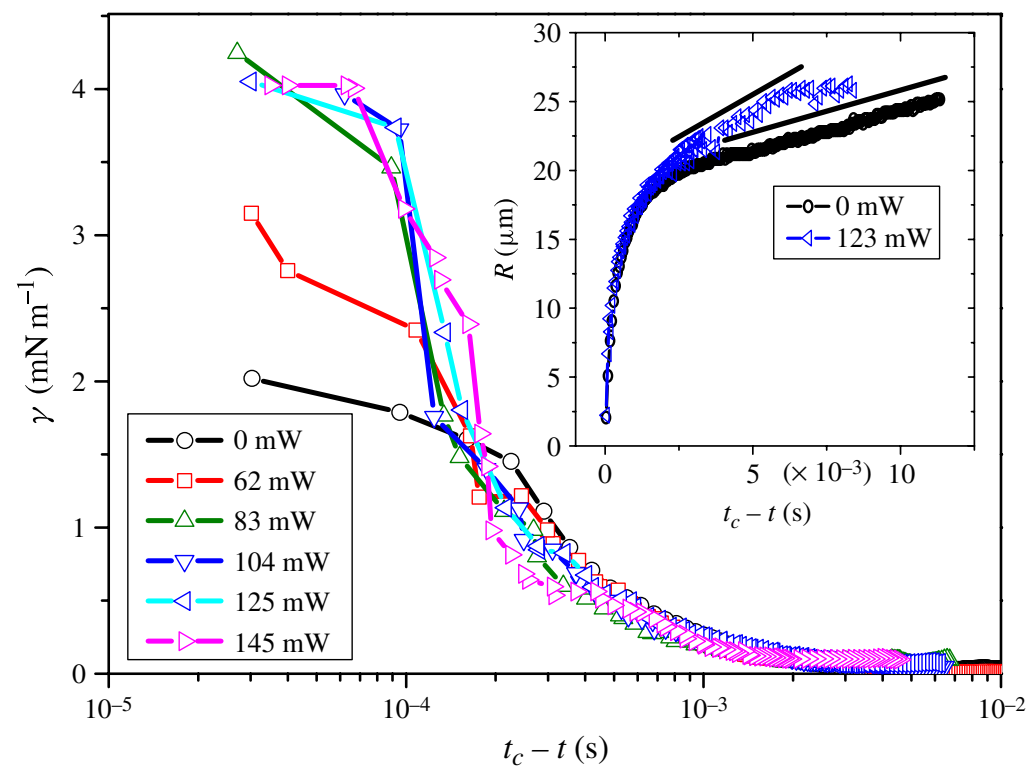

Figure 5. (Colour online) Pinch-off in microfluidic channels: drop formation and jet breakup under the action of a laser beam. The thinning dynamics is shown in the inset (the full lines indicate the initial linear regime) and the dynamic interfacial tension is shown in the main figure. When a laser beam is used to break the jet, the final tensions seem to increase with the increase of laser power before saturating. Imposed flow rates are: $Q_{o}=1 \mu 1 \mathrm{~min}^{-1}$ (oil) and $Q_{w}=0.2 \mu \mathrm{min}^{-1}$ (water) for breakup in a cross-shaped junction, and $Q_{o}=1.2 \mu 1 \mathrm{~min}^{-1}$ and $Q_{w}=4 \mu 1 \mathrm{~min}^{-1}$ for laser-induced breakup.

magnitude and that different experiments give a roughly similar increase versus time despite the differences in producing pinch-off (with or without a laser beam and with and without local heating). The initial values of the tension vary from run to run in this system. As noted above, this system may be very sensitive to the history of the interface, a fact which we attribute to the presence of two surfactants, one in each phase. The equilibrium in this system therefore depends on how the two surfactants cover the surface. Nonetheless different experiments - pendant drops, dripping in a cross-channel, and laser-induced jet breakup - give interfacial tension values near $0.1 \mathrm{mN} \mathrm{m}^{-1}$ at the initial instants and which increase by an order of magnitude as the rupture point is approached. It should be noted here that the laser-initiated pinch-off seems to produce higher final interfacial tensions than the simple pinch-off case in a cross-shaped junction or in the dripping experiment, which is likely to be due to additional thermocapillary stresses due to heating.

\section{Conclusion}

In conclusion, different systems have been used to study the rupture of fluid necks resulting from the detachment of small drops of fluid in the presence of surfactants. The use of such systems has allowed us to probe the effect of the surfactant on the different regimes of the rupture process, namely the inertial and viscous regimes. In all cases, the effect of the surfactant on the thinning dynamics can be described as an increase of the local interfacial tension at the location of the minimum neck radius when pinch-off is approached. The thinning dynamics can therefore be recast using a 
time-varying surface tension. This surface tension starts at values near its equilibrium value and increases as the rupture is approached. This increase is a measure of the depletion of surfactant from the pinch-off location as found in numerical and theoretical studies of pinch-off in the presence of surfactants. In the final instants before breakup the surface tensions remain below those of the bare interfaces except for the very low concentrations of surfactant. Our procedure allows us to estimate dynamic interfacial tensions in a new configuration: that of a stretching neck starting from a fully, or partially, covered interface. The usual way is to start from a bare surface and measure the dynamic tension as adsorption takes place.

\section{REFERENCES}

Amarouchene, Y., Bonn, D., Meunier, J. \& Kellay, H. 2001 Inhibition of the finite-time singularity during droplet fission of a polymeric fluid. Phys. Rev. Lett. 86, 3558.

Ambravaneswaran, B. \& Basaran, O. A. 1999 Effects of insoluble surfactants on the nonlinear deformation and breakup of stretching liquid bridges. Phys. Fluids 11, 997.

Aytouna, M., Bartolo, D., Wegdam, G., Bonn, D. \& RafaÏ, S. 2010 Impact dynamics of surfactant laden drops: dynamic surface tension effects. Exp. Fluids 48, 49.

Binks, B. P., Cho, W. G., Fletcher, P. D. I. \& Petsev, D. N. 2000 Stability of oil-in-water emulsions in a low interfacial tension system. Langmuir 16, 1025.

Christov, N. C., Danov, K. D., Kralchevsky, P. A., Ananthapadmanabhan, K. P. \& LIPS, A. 2006 Maximum bubble pressure method: universal surface age and transport mechanisms in surfactant solutions. Langmuir 22, 7528.

Cohen, I., Brenner, M. P., Eggers, J. \& Nagel, S. R. 1999 Two fluid drop snap-off problem: experiments and theory. Phys. Rev. Lett. 83, 1147.

Cohen, I. \& NAGEL, S. R. 2001 Testing for scaling behaviour dependence on geometrical and fluid parameters in the two fluid drop snap-off problem. Phys. Fluids 13, 3533.

Craster, R. V., Matar, O. K. \& Papageorgiou, D. T. 2002 Pinchoff and satellite formation in surfactant covered viscous threads. Phys. Fluids 14, 1364.

Craster, R. V., Matar, O. K. \& Papageorgiou, D. T. 2009 Breakup of surfactant-laden jets above the critical micelle concentration. J. Fluid Mech. 629, 195.

Delville, J. P., Robert de Saint Vincent, M., Schroll, R. D., Chraïbi, H., Issenmann, B., Wunenburger, R., Lasseux, D., Zhang, W. W. \& Brasselet, E. 2009 Laser microfluidics: fluid actuation by light. J. Opt. A: Pure Appl. Opt. 11, 034015.

Doshi, P., Suryo, R., Yildirim, O. E., McKinley, G. H. \& Basaran, O. A. 2003 Scaling in pinch-off of generalized Newtonian fluids. J. Non-Newtonian Fluid Mech. 113, 1-27.

Eggers, J. 1993 Universal pinching of 3D axisymmetric free-surface flow. Phys. Rev. Lett. 71, 3458 .

EgGers, J. 1997 Nonlinear dynamics and breakup of free-surface flows. Rev. Mod. Phys. 69, 865.

Eggers, J. \& Villermaux, E. 2008 Physics of liquid jets. Rep. Prog. Phys. 71, 036601.

Jin, F., Gupta, N. R. \& Stebe, K. J. 2006 The detachment of a viscous drop in a viscous solution in the presence of a soluble surfactant. Phys. Fluids 18, 022103.

Liao, Y. C., Franses, E. I. \& Basaran, O. A. 2006 Deformation and breakup of a stretching liquid bridge covered with an insoluble surfactant monolayer. Phys. Fluids 18, 022101.

Liao, Y. C., Subramani, H. J., Franses, E. I. \& Basaran, O. A. 2004 Effects of soluble surfactants on the deformation and breakup of stretching liquid bridges. Langmuir 20, 9926.

Lister, J. R. \& Stone, H. A. 1998 Capillary breakup of a viscous thread surrounded by another viscous fluid. Phys. Fluids 10, 2758.

McGough, P. T. \& Basaran, O. A. 2006 Repeated formation of fluid threads in breakup of a surfactant-covered jet. Phys. Rev. Lett. 96, 054502.

Mitani, S. \& SAKAI, K. 2002 Measurement of ultralow interfacial tension with a laser interface manipulation technique. Phys. Rev. E 66, 031604.

Papageorgiou, D. T. 1995 On the breakup of viscous liquid threads. Phys. Fluids 7, 1529. 
RENARDY, M. \& RENARDY, Y. 2004 Similarity solutions for breakup of jets of power law fluids. J. Non-Newtonian Fluid Mech. 122, 303.

Roché, M., Aytouna, M., Bonn, D. \& Kellay, H. 2009 Effect of surface tension variations on the pinch-off behavior of small fluid drops in the presence of surfactants. Phys. Rev. Lett. 103, 264501.

Rothert, A., Richter, R. \& Rehberg, I. 2001 Transition from symmetric to asymmetric scaling function before drop pinch-off. Phys. Rev. Lett. 87, 084501.

Savage, J. R., Caggioni, M., Spicer, P. T. \& Cohen, I. 2010 Partial universality: pinch-off dynamics in fluids with smectic liquid crystalline order. Soft Matt. 6, 892.

Timmermans, M. L. E. \& Lister, J. R. 2002 The effect of surfactant on the stability of a liquid thread. J. Fluid Mech. 459, 289.

Wagner, C., Amarouchene, Y., Bonn, D. \& Eggers, J. 2005 Droplet detachment and satellite bead formation in viscoelastic fluids. Phys. Rev. Lett. 95, 164504.

XU, Q., LiaO, Y. C. \& Basaran, O. A. 2007 Can surfactant be present at pinch-off of a liquid filament? Phys. Rev. Lett. 98, 054503.

ZHANG, W. W. \& Lister, J. R. 1999 Similarity solutions for capillary pinch-off in fluids of differing viscosity. Phys. Rev. Lett. 83, 1151. 